\title{
Renoprotective Effect of Sulphate Polysaccharide from Brown Algae on Ethylene Glycol-Induced Renal Damage in Rats
}

\author{
Hui Huang, ${ }^{a, b}$ Junwen Wang, ${ }^{a, b}$ Xueyan Li, ${ }^{a, b}$ Wenhui Wu, ${ }^{a, b, c, d}$ Kejin Shi, ${ }^{*, e}$ and Chaoyan Zhang ${ }^{\star, a, b, c, d}$ \\ ${ }^{a}$ College of Food Science and Technology, Institutes of Marine Sciences, Shanghai Ocean University, \\ Shanghai 201306, China \\ ${ }^{b}$ National Research Center for Freshwater Aquatic Products Processing Technology (Shanghai), \\ Shanghai 201306, China \\ ${ }^{c}$ Shanghai Aquatic Products Processing and Storage Engineering Technology Research Center, \\ Shanghai 201306, China \\ ${ }^{d}$ Laboratory of Quality and Safety of Aquatic Products Storage and Safety in Ministry of Agriculture, \\ Shanghai 201306, China \\ ${ }^{e}$ Sichuan Institute of antibiotics industry, Chengdu University, Chengdu, Sichuan 610052, China
}

Email: shikejin1228@163.com (K. S.), chyzhang@shou.edu.cn (C. Z.)

\begin{abstract}
The protective effect of the polysaccharides on ethylene glycol induced kidney impairment has been explored. Polysaccharides have been extracted from Sargassum graminifolium, which belongs to brown algae. Three different doses of polysaccharides were used for rats with kidney stones by ethylene glycol feeding. Then, urinary biochemistry parameters, renal function factors including blood urea nitrogen (BUN) and serum creatinine (Scr) levels were detected. Besides, interleukin-6 (IL-6) and tumor necrosis factor a (TNF- $\alpha$ ) in kidney tissue were elevated, which belong to proinflammatory cytokines. Moreover, kidney histopathological sections were examined. The results illustrated that polysaccharides not only increased calcium level but also decreased oxalate, Scr, and BUN levels. In addition, the levels of IL-6 and TNF- $\alpha$ were reduced. Moreover, renal microscopic analysis showed polysaccharides treatment alleviated observably calcium oxalate deposits in the kidney tissue. The results indicated that the renoprotective mechanism of SGP may be attributed to suppression of inflammation, reducing the growth of urinary stones and improving kidney function in vivo, which explained SGP through multiple ways to protect kidney cells from damaging of hyperoxaluria rats.
\end{abstract}

Keywords brown algae, polysaccharides, hyperoxaluric, urinary stones, inflammation, antioxidants

\section{Introduction}

As we all know, seaweed from marine is the important bioresource because of its abundant bioactivities. The activities of brown algae, red algae and green algae have been researched worldwide for many years. The active substances from these algae possess wide applications. Among them, the most important active substance is a series of sulfated polysaccharides. Many reports showed that sulfated polysaccharides are of great importance due to their bioactivities such as inhibiting lipase activity, inhibiting amyloid-beta-induced toxicity, immunoregulation, and antiviral activities, etc. ${ }^{[1-8]}$

Urinary stone is a common disease with high recurrence rate in the world mainly caused by pathological mineralization in vivo. The incidence and recurrence rate of urolithiasis are generally on the rise. The etiology and pathogenesis of $\mathrm{CaO}_{x}$ calculi is complex. Much work needs to be performed to control the incidence and recurrence rate of $\mathrm{CaO}_{x}{ }^{[9-11]}$ Recently, the relationship between oxidative stress/cell damage and the occurrence of $\mathrm{CaO}_{x}$ stones has attracted great attention. Exposure of kidney cells to $\mathrm{CaO}_{x}$ crystals results in the production of reactive oxygen species (ROS), which is involved in a variety of signaling pathways. This leads to oxidative stress in kidney cells, and then further causes renal injury and inflammation, which play a key role in the formation of urinary stones. ${ }^{[12-16]}$ Clinical research indicates that fetoglobulin $A$ is found in urine and kidney of patients with stones. Fetoprotein A is an inflammation-related glycoprotein, which can be anti-inflammatory and directly inhibit macrophages. At the same time, inflammatory cytokines (IL-6, TNF- $\alpha$ ) also affect the synthesis and secretion of this protein. ${ }^{[17-19]}$

Sargassum C. Agardh belongs to brown algae, and it distributes in tropical and temperate regions. There are more than 250 species in the world including 130 species recorded in China. Sargassum grasss is the traditional source of medicinal seaweed in China. According to the records, 15 species of Sargassum can be utilized as medicinal seaweed including Sargassum grass. ${ }^{[20,21]}$

Our previous works showed that polysaccharides of Sargassumgrasss (SGP) had excellent antioxidant activity by measuring SGP's reducing power, scavenging superoxide anion free radicals and inhibiting DPPH free radicals. On one hand, it inhibited calcium oxalate crystallization in vitro; on the other hand, it protected mitochondrial function of rats with kidney stones in vivo. ${ }^{[22,23]}$

Currently, urinary parameters, immune modulator and renal function about the mechanisms of SGP are not investigated. In this paper, we will explore the possible mechanism in vivo from multiple aspects with renal function, immune modulator and reducing $\mathrm{CaO}_{x}$ crystal deposition. 
Table 1 The rats divided group in experimental hyperoxaluria

\begin{tabular}{|c|c|}
\hline Groups & Treatment \\
\hline Group A & Drinking water from the 1 st day up to the end of the experimental period \\
\hline Group B & $E G+A C$ in drinking water for $7 \mathrm{~d}$ \\
\hline Group C & $E G+A C$ in drinking water for $7 \mathrm{~d}+\mathrm{SGP} 400 \mathrm{mg} / \mathrm{kg}$ from the 8 th day up to the end of the experimental period \\
\hline Group D & $\mathrm{EG}+\mathrm{AC}$ in drinking water for $7 \mathrm{~d}+\mathrm{SGP} 100 \mathrm{mg} / \mathrm{kg}$ from the 8 th day up to the end of the experimental period \\
\hline Group E & $E G+A C$ in drinking water for $7 \mathrm{~d}+\mathrm{SGP} 25 \mathrm{mg} / \mathrm{kg}$ from the 8th day up to the end of the experimental period \\
\hline
\end{tabular}

\section{Experimental}

\section{Reagents}

Reagents and kits used for histological preparations or assay of urine parameters, renal function were purchased from Nanjing Jian Cheng Bio Inst. All the other chemical reagents applied were of analytical grade purchased from Shanghai Guo Yao Group Chemical Reagent Co., Ltd.

The more information about SGP such as extract and molecular weight, structure and sulphuric acid content has been described in our previous achievement. ${ }^{[23]}$

\section{Animals}

Before the experiment, the male Wistar rats with body weight at $200 \pm 20 \mathrm{~g}$ were domesticated in cages for $7 \mathrm{~d}$ at temperature of $22 \pm 2{ }^{\circ} \mathrm{C}$, and then they were placed in a controlled light-dark cycle for $12 \mathrm{~h}$. All rats were fed in a standard diet.

\section{Ethylene glycol-induced renal damage in rats}

The male Wistar rats were randomly divided into 5 groups, 6 in each group. The specific groups are shown in Table 1. It is very important to establish and validate an experimental animal model of kidney stones in order to explore a renoprotective effect of SGP on ethylene glycol-induced renal damage. At present, ethylene glycol is most commonly used for construction of a kidney stone model due to its good repeatability and relatively stability. The principle is that ethylene glycol is the precursor of oxalic acid, which can be converted into oxalic acid by metabolism in vivo. In this modeling scheme, ammonium chloride acts as acidifying urine and contributes to the formation of kidney stones. ${ }^{[24,25]}$ In this model, the concentration of ethylene glycol (EG) was $1 \%[V / V]$ and ammonium chloride (AC)) was $1 \%[\mathrm{w} / \mathrm{V}]$.

\section{Collection and analysis of urine}

The male Wistar rats were placed in individual metabolic cages, whose $24 \mathrm{~h}$ urine samples were gathered every day. Then, urine volumes of $24 \mathrm{~h}$ were recorded, the urine of $\mathrm{pH}$ was measured. After adding sodium and sodium corrosion, the urine was collected by centrifuge (3000 rpm, $15 \mathrm{~min})$. Then, the contents of oxalate and calcium ions in the supernatant were examined. Calcium and oxalate were tested according to instructions of kits, which were bought from Nanjing Jian Cheng Bio Inst.

\section{Assessment of renal function}

The assay of Scr is based on a product of orange, which is formed by creatinine clearance of blood protein in supernatant with picric acid in Jaffe reaction. The assay of BUN is based on Fearon reaction, which means urea in serum can be condensed into a red compound after azeotropic with diacetyl-oxime in the acidic environment of urea nitrogen reagent. The shade of the color is proportional to the self-content of urea in serum and the content of urea nitrogen in serum can be measured by colorimetric comparison with the same treated urea nitrogen standard solution. The assay of Scr and BUN were tested according to instructions of kits. The kits were bought from Nanjing Jian Cheng Bio Inst.

\section{The expression levels of TNF-a and IL- 6 in renal}

TNF-a and IL- 6 were tested using kits from Nanjing Jian Cheng Bio Inst, and the protocols were all followed according to the manufacturer's instructions in the kit.

\section{Histopathological studies}

The rats were sacrificed after anesthesia and two kidneys were removed from each rat. Cut the left kidney, take $1.0 \mathrm{~cm} \mathrm{x}$ $1.0 \mathrm{~cm} \times 0.3 \mathrm{~cm}$ size of the tissue block, quickly put into $10 \%$ formaldehyde solution fixed. The fixed time is $48 \mathrm{~h}$. This operation can make tissue and cell denaturation of the protein coagulation, to prevent cell death after autolysis and bacterial decomposition, thereby keeping the cell originally morphology. Then, the kidney tissue was treated with a series of different concentration alcohols and xylene, embedded in paraffin, sectioned and stained with Haematoxylin and Eosin for histopathological examination. Hematoxylin (HE) staining was used to observe the histopathological changes of right kidney sections under polarizing microscope (MOTIC BA200).

\section{Statistical analysis}

Data were expressed as mean \pm standard deviation. One-way ANOVA with post hoc Dunnett's test or by Student's $t$-test was applied to determine differences among different groups using the SPSS software package for Windows. Significance has been showed in the following table and figures.

\section{Results and Discussion}

\section{Effect of SGP on urinary parameters}

The effect of various doses of SGP on rat urine volume, oxalate, $\mathrm{Ca}^{2+}$ and $\mathrm{pH}$ is shown in Table 2. It was found that urine volume and oxalate increased significantly between Group B and Group A; however, the amount of calcium in urine decreased significantly $(P<0.01)$. Administration of SGP to rats with kidney stones was able to increase $\mathrm{Ca}^{2+}$ levels. Groups $\mathrm{C}$, $\mathrm{D}$ and $\mathrm{E}$ increased the $\mathrm{Ca}^{2+}$ level significantly compared with Group B. Besides this, Groups C and D decreased the oxalate level evidently $(P<0.01)$. However, there was no significant difference of urine volume and $\mathrm{pH}$ among the groups rats administrated of SGP and Group B. During the experiment, there was no significant change in the weight of rats in each group.

\section{Effect of SGP on renal detection index}

Both serum creatinine (Scr) and blood urea nitrogen (BUN) 
Table 2 The urinary biochemistry results of each group rat in experimental hyperoxaluria

\begin{tabular}{cccccc}
\hline Parameters & Group A & Group B & Group C & Group D & Group E \\
\hline Urine $(\mathrm{mL} / 24 \mathrm{~h})$ & $16.10 \pm 0.70$ & $38.15 \pm 3.57^{\#}$ & $43.83 \pm 1.62$ & $42.10 \pm 1.23$ & $42.70 \pm 2.56$ \\
Oxalate $(\mu \mathrm{mol} / 24 \mathrm{~h})$ & $20.30 \pm 6.34$ & $75.13 \pm 4.57^{\# \#}$ & $34.51 \pm 3.85^{\star *}$ & $46.67 \pm 2.18^{\star *}$ & $63.55 \pm 5.83$ \\
$\mathrm{Ca}^{2+}(\mu \mathrm{mol} / 24 \mathrm{~h})$ & 15.193 .84 & $4.96 \pm 0.91^{\# \#}$ & $12.77 \pm 1.20^{\star *}$ & $8.89 \pm 0.86^{\star *}$ & $6.18 \pm 0.57^{\star}$ \\
$\mathrm{pH}$ & $6.62 \pm 0.13$ & $6.27 \pm 0.24$ & $6.56 \pm 0.17$ & $6.45 \pm 0.21$ & $6.33 \pm 0.19$ \\
\hline
\end{tabular}

\# $P<0.05,{ }^{\# \#} P<0.01$ (vs. Group A); ${ }^{*} P<0.05,{ }^{* *} P<0.01$ (vs. Group B).
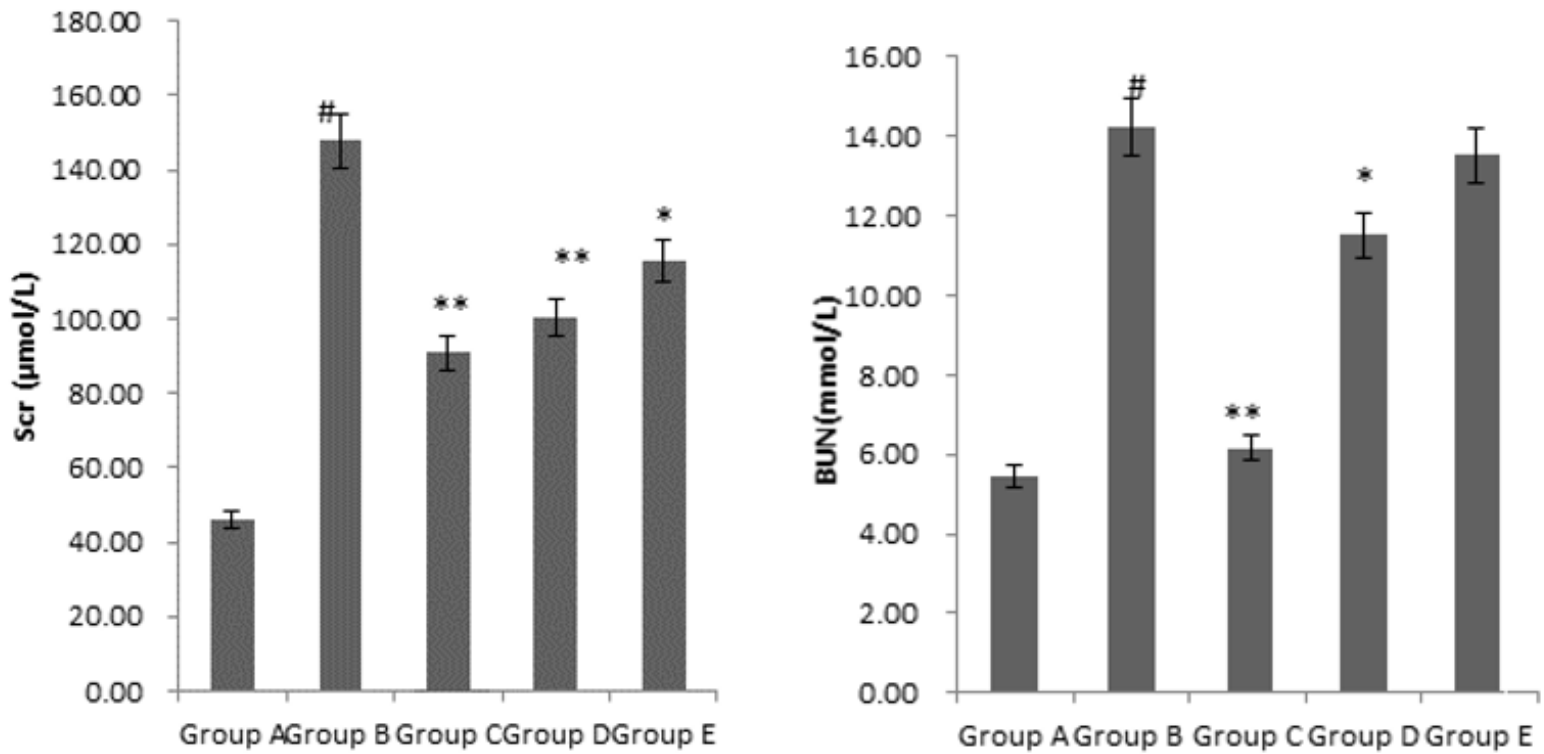

Figure 1 BUN and Scr results of each group rats in experimental hyperoxaluria. ${ }^{\#} P<0.05,{ }^{\# \#} P<0.01$ (vs. Group A); ${ }^{*} P<0.05$, ${ }^{* *} P<0.01$ (vs. Group B).

contents as the renal detection index are measured at the same time in the clinical. It means that the kidney function has serious damage when the indexes are higher than the normal values. Figure 1 shows BUN and Scr contents of experimental rats groups. It was found that BUN and Scr activity of Group B increased evidently in comparison to Group A, indicating that ethylene glycol had impaired renal function of the rats. Compared with Group B, the serum BUN and Scr contents in Group $C$ were significantly decreased $(P<0.01)$. In addition, Group D lowered BUN level evidently $(P<0.05)$, too.

\section{Serum levels of proinflammatory cytokines}

The proinflammatory cytokines include tumor necrosis factor alpha (TNF- $\alpha$ ) and interleukin-6 (IL-6), and their levels can reflect the renal tubular injury and inflammatory conditions. Figure 2 showed that the contents of TNF- $\alpha$ and IL-6 in Group B increased compared with Group A. After administration of SGP, the levels of TNF- $\alpha$ and IL-6 in Groups C-E were lowered compared with Group B. Groups C and D lowered the levels of TNF- and IL-6 evidently.

\section{Calcium oxalate deposits in the kidney section}

The morphology of the crystals in renal tissues are shown in Figures $3 a-3 e$.

Extensive white $\mathrm{CaO}_{x}$ crystals deposition were observed in Figures $3 a-3 d$. Moreover, large $\mathrm{CaO}_{x}$ crystals were detected in most of the renal tubules in Figure $3 d$, which belong to ethylene glycol induced hyperoxaluric rats. Interestingly, white
$\mathrm{CaO}_{x}$ crystals became smaller from tens of microns down to a few microns especially with the SGP from a dose of $25 \mathrm{mg} / \mathrm{kg}$ increased to $400 \mathrm{mg} / \mathrm{kg}$. In Figure 1e (normal control group), no obvious $\mathrm{CaO}_{x}$ crystal was observed.

Figure $3 f$ showed a lot of $\mathrm{CaO}_{x}$ crystals in the urinary of ethylene glycol induced hyperoxaluricrats, which were prominent edges and typical hexahedral $\mathrm{CaO}_{x}$ crystals. This also indicates ethylene glycol induced renal injury and $\mathrm{CaO}_{x}$ crystals deposition in male SD rats.

\section{Conclusions}

Kidney stone is widespread in the world, whose formation causes a chronic disease. Recent studies showed that ethylene glycol not only induced the deposition of $\mathrm{CaO}_{x}$ crystals in the kidney but also could lead to kidney damage and inflammation. ${ }^{[26-28]}$ Khan et al. reported that high oxalate and uric acid levels could lead to $\mathrm{CaO}_{x}$, which can damage cells and induce inflammation and secondary fibrosis, leading to chronic end-stage kidney disease at last. ${ }^{[15]}$ Their research showed that crystal deposition was a key, which could induce damage and inflammation. Thus, crystal retention played a great role in the urinary stone process. ${ }^{[29]}$ Then, $\mathrm{CaO}_{x}$ was related to oxidative stress and TNF- $\alpha$ and IL- 6 expression, which may result in high recurrence rate of urinary stone. ${ }^{[30-32]}$

In Bhadja's review, ${ }^{[33]}$ the action mechanism of algae polysaccharides on $\mathrm{CaO}_{x}$ renal stone was revealed in detail in Figure 4 . The sequential schematic for the inhibitory mecha- 

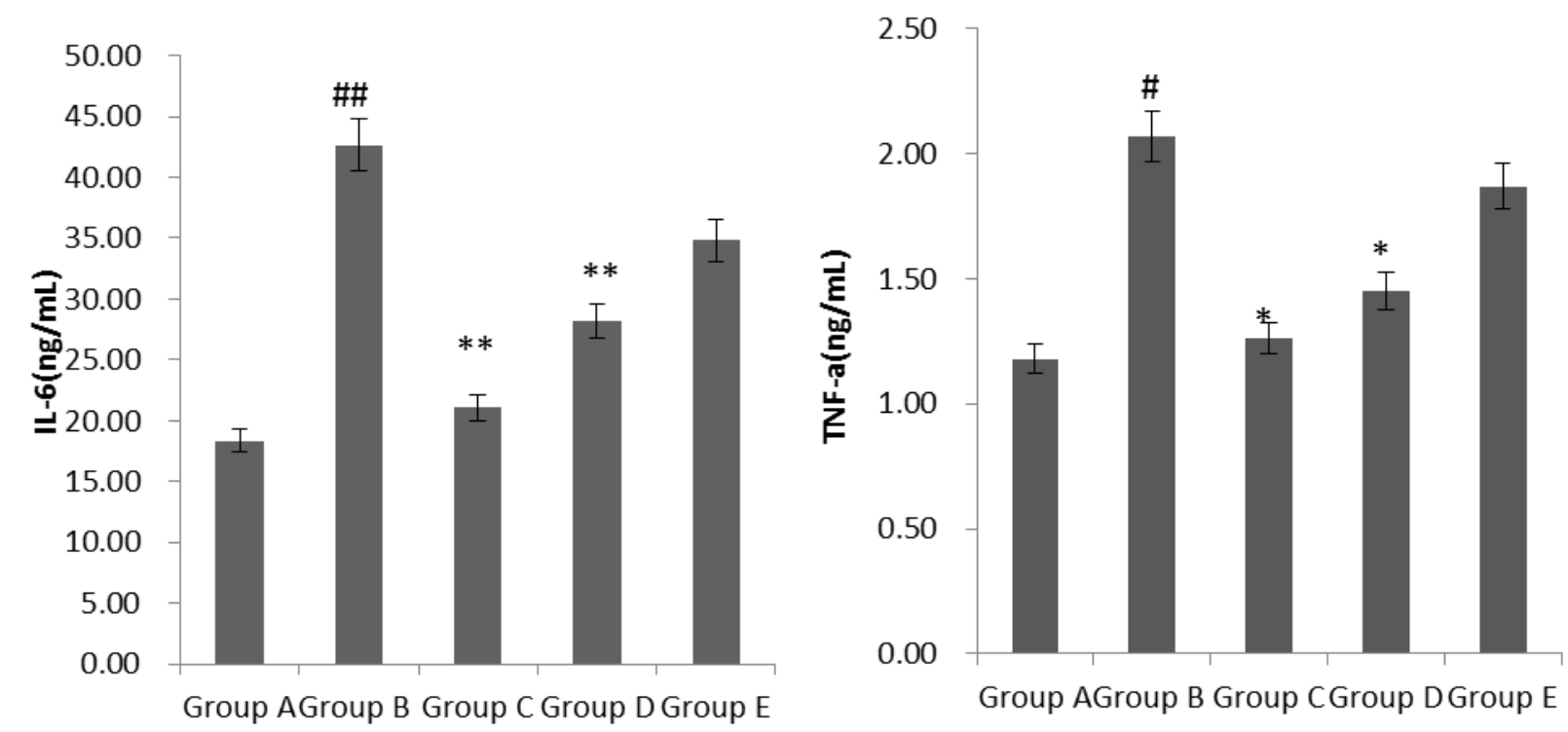

Figure 2 TNF- $\alpha$ and IL-6 results of each group rats in experimental hyperoxaluria. \# $P<0.05$, \#\# $P<0.01$ (vs. Group A); $P<0.05$, ${ }^{* *} P<0.01$ (vs. Group B).
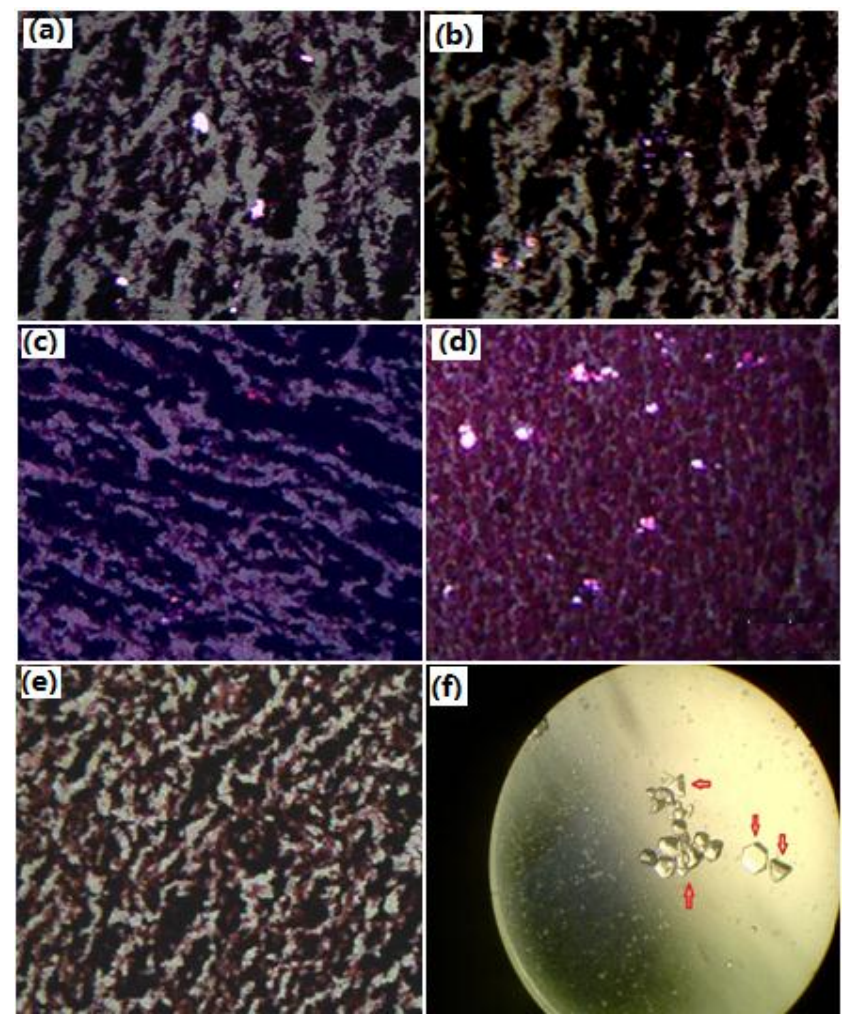

Figure 3 Crystalline formations in the rat kidney ( $a-e$, polarizing microscope, magnification $\times 40$ ). The morphology of the crystals in renal tissues is shown in a-e.

nism of algae polysaccharides at each step of $\mathrm{CaO}_{x}$ stone formation can be seen. Also, sulfated polysaccharides in seaweeds have a large number of negatively charged groups such as sulfate, carboxyl, and hydroxyl groups. These anions have a strong ability to interact with calcium ions. ${ }^{[34]}$ In brief, sulfated polysaccharides from the algae protect and repair the renal cells by blocking or inhibiting the injury pathways and increasing the antioxidant capacity of cells. The algae polysaccharides are promising molecules due to their role in

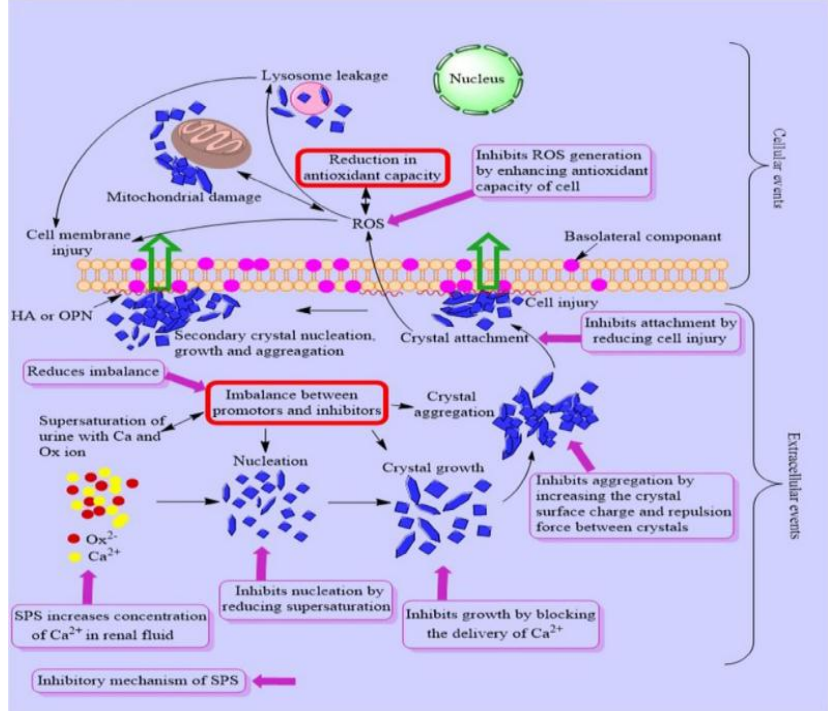

Figure 4 The sequential schematic for the inhibitory mechanism of seaweed polysaccharides at each step of $\mathrm{CaO}_{x}$ stone formation. ${ }^{[33]}$

renal stone prevention.

Our team disclosed that sulfated polysaccharides of Sargassumgrasss possessed excellent antioxidant activity. It inhibited calcium oxalate crystallization in vitro, furthermore, it protected mitochondrial function of rats with kidney stones in vivo. $^{[2,23]}$ Interestingly, the treatment with SGP increased the $\mathrm{Ca}^{2+}$ level, decreased oxalate and oxalate deposits, reduced the TNF-a and IL-6 levels, and lessened the SCr and BUN levels. Strikingly, the results showed that the renoprotective effect of SGP was due to inflammatory inhibition and reducing oxalate deposits as well as improving renal function. Together with excellent antioxidant activity SGP in vitro and in vivo, SGP can be used as a promising therapeutic agent for ethylene glycol-induced renal injury and preventing currency of urinary stone. 


\section{Acknowledgement}

This work was funded by the Science and Technology Development Fund of Shanghai Ocean University (No. A2-0209-14-200069) and the Doctoral Research Fund of Shanghai Ocean University (No. A1-0209-13-0105394).

\section{Author Contributions}

The conceptualization was performed by Dr. Chaoyan Zhang and Dr. Wenhui Wu, the methodology was carried out by Junwen Wang and Xueyan Li, the software was from Dr. Jinshi $\mathrm{Ke}$, the investigation was carried out by Junwen Wang and Xueyan $\mathrm{Li}$, the resource was from $\mathrm{Dr}$. Jinshi $\mathrm{Ke}$, the data curation was performed by Hui Huang, and the original draft was prepared by Hui Huang. This paper was written/reviewed/ edited by Dr. Chaoyan Zhang, the supervision of this work was by $\mathrm{Dr}$. Wenhui Wu, the project administration was performed by Dr. Chaoyan Zhang, and the fund acquisition was carried out by Dr. Chaoyan Zhang.

\section{Conflict of Interest}

The authors declare no conflict of interest.

Copyright @ 2020 Hui Huang, Junwen Wang, Xueyan Li, Wenhui Wu, Kejin Shi, and Chaoyan Zhang. This article is an open access article distributed under the terms and conditions of the Creative Commons Attribution (CC BY) license (http://creativecommons.org/licenses/by/ 4.0/). The use, distribution or reproduction in other forums is permitted, provided the original author(s) or licensor are credited and that the original publication in this journal is cited, in accordance with accepted academic practice. No use, distribution or reproduction is permitted which does not comply with these terms.

\section{References}

[1] Karnjanapratum, S.; You, S. Molecular characteristics of sulfated polysaccharides from Monostroma nitidum and their in vitro anticancer and immunomodulatory activities. Int. J. Biol. Macromol. 2010, 2, 311-318.

[2] Zhang, Z.; Zhang, Q.; Wang, J.; Zhang, H.; Niu, X.; Li, P. Preparation of the different derivatives of the low-molecular-weight porphyran from Porphyra haitanensis and their antioxidant activities in vitro. Int. J. Biol. Macromol. 2009, 1, 22-26.

[3] Rajeswari, A.; Varalakshmi, P. Low molecular weight heparin protection against oxalate-induced oxidative renal insult. Clin. Chim. Acta 2006, 1, 108-114.

[4] Wijesekara, I.; Pangestuti, R.; Kim, S.K. Biological activities and potential health benefits of sulfated polysaccharides derived from marine algae. Carbohydr. Polym. 2011, 1, 14-21.

[5] Matsuda, M.; Okutani, K.; Shigeta, S. In vitro antiviral activities of sulfated polysaccharides from a marine microalga (Cochlodinium polykrikoides) against human immunodeficiency virus and other enveloped viruses. Int. J. Biol. Macromol. 1995, 5, 293-297.

[6] Kardošová, A.; Machová, E. Antioxidant activity of medicinal plant polysaccharides. Fitoterapia 2006, 77, 367-373.

[7] Li, S.; Li, J. H.; Zhi, Z. J. Macromolecular properties and hypolipidemic effects of four sulfated polysaccharides from sea cucumbers Carbohydr. Carbohydr. Polym. 2017, 173, 330-337.

[8] Wang. X. L.; Yi, K. X.; Zhao, Y. Fucoidan inhibits amyloid-betainducedtoxicityin transgenic Caenorhabditis elegans by reducing the accumulation of amyloid-beta and decreasing the production of reactive oxygen species. Food Funct. 2018, 9, 552-560.

[9] Borghi, L.; Meschi, T.; Guerra, A.; Bergamaschi, E.; Mutti, A.; Novarini, A. Effects of urinary macromolecules on the nucleation of calcium oxalate in idiopathic stone formers and healthy controls. Clin. Chim. Acta 1995, 1, 1-11.

[10] Edyvane, K. A.; Hibberd, C. M.; Harnett, R. M.; Marshall, V. R.;
Ryall, R. L. Macromolecules inhibit calcium oxalate crystal growth and aggregation in whole human urine. Clin. Chim. Acta 1987, 3, 329-338.

[11] Liu, J.; Wang, T.; Chen, J.; Wang, S.; Ye, Z. Decreased inhibitory activity of prothrombin to calcium oxalate crystallization by specific chemical modification of its gamma-carboxyglutamic acid residues. Urology 2006, 1, 201-203.

[12] Hackett, R. L.; Shevock, P. N.; Khan, S. R. Madin-Darby canine kidney cells are injured by exposure to oxalate and to calcium oxalate crystals. Urol. Res. 1994, 22, 197.

[13] Koul, H.; Kenington, L.; Honeyman, T.; Jonassen, J.; Menon, M.; Scheid, C. R. Activation of the c-myc gene mediates the mitogenic effects of Ox in LLC-PK1 cells, a line of renal epithelial cells. Kidney Int. 1996, 50, 1525-1530.

[14] Bashir, S.; Gilani, A. H. Antiurolithic effect of Bergenia ligulata rhizome: An explanation of the underlying mechanisms. J. Ethnopharmacol. 2009, 1, 106-116.

[15] Khans, R. Crystal-induced inflammation of the kidneys: results from human studies, animal models, and tissue-culture studies. Clin. Exp. Nephrol. 2004, 8, 75-88.

[16] Guerne, P. A.; Terkeltaub, R.; uraw, B.; Lotz, M. Inflammatory microcrystals stimulate interleukin- 6 production and secretion by human monocytes and synoviocytes. Arthritis Rheum. 1989, 32, 1443-1452.

[17] Volker, V.; Tanya, N. M. Functions of TNF and its Receptors in Renal Disease: Distinct Roles in Inflammatory Tissue Injury and Immune Regulation. Semin. Nephrol. 2007, 3, 286-308.

[18] Di Paola, R.; Genovese, T.; Impellizzeri, D.; Ahmad, A.; Cuzzocrea S.; Esposito, E. The renal injury and inflammation caused by ischemia-reperfusion are reduced by genetic inhibition of TNFaR1: A comparison with infliximab treatment. Eur. J. Pharmacol. 2013, 700, 134-146.

[19] Wu, Y. X.; Li. C. Y.; Deng, Y. L. Patients with nephrolithiasis had lower fetuin-A protein level in urine and renal tissue. Urolithiasis 2014, 42, 29-37.

[20] Wang, J.; Zhang, Q. B.; Zhang, Z. S.; Zhang, H.; Niu, X. Z. Structural studies on a novel fucogalactan sulfate extracted from the brown seaweed Laminaria japonica. Int. J. Biol. Macromol. 2010, 47, 126-131.

[21] Samee, H.; Li, Z. X.; Lin, H.; Khalid, J.; Wang, B. P. In vivo study of antiallergenicity of ethanol extracts from Sargassum tenerrimum, Sargassum cervicorne and Sargassum graminifolium turn. Eur. Food Res. Technol. 2009, 229, 435-441.

[22] Zhang, C. Y.; Wu, W. H.; Lan, M. B. Antioxidant properties of polysaccharide from the brown Seaweed Sargassum graminifolium (Turn.), and its effects on calcium oxalate crystallization. Mar. Drugs 2012, 10, 119-130.

[23] Zhang, C. Y.; Kong T.; Wu, W. H.; Lan, M. B. The Protection of Polysaccharide from the Brown Seaweed Sargassum graminifolium against Ethylene Glycol-Induced Mitochondrial Damage. Mar. Drugs 2013, 11, 870-880.

[24] Orhan, N.; Onaran, M.; Şen, I.; Aslan, M. Preventive treatment of calcium oxalate crystal deposition with immortal flowers. J. Ethnopharmacol. 2015, 163, 60-67.

[25] Yuen, J. W. M.; Gohel, M. D. I.; Poon, N. W.; Shum, D. K. Y.; Tam, P. C.; $\mathrm{Au}, \mathrm{D}$. W. T. The initial and subsequent inflammatory events during calcium oxalate lithiasis. Clin. Chim. Acta 2010, 411, 1018-1026.

[26] Parveen, K. G.; Santosh, K. V.; Anil, K. S. Optimization of extraction protocol of Parmelia perlata and its validation for protective effects against oxalate-induced renal injury in NRK-52E cells. J. Herb. Med. 2018, 12, 79-87.

[27] :Mulay, S. R.; Kulkarni, O. P.; Rupanagudi, K. V.; Migliorini, A.; Darisipudi, M. N.; Vilaysane, A.; Muruve, D.; Shi, Y.; Munro, F.; Liapis, H.; Anders, H. J. Calcium oxalate crystals induce renal inflammation by NLRP3-mediated IL-1beta secretion. J. Clin. Invest. 2013, 123, 236-246. 
[28] Khan, S. R. Reactive oxygen species, inflammation and calcium oxalate nephrolithiasis. Transl. Androl. Urol. 2014, 3, 256-276.

[29] Joshi, S.; Wang, W.; Peck, A. B.; Khan, S. R. Activation of the NLRP3 Inflammasome in Association with Calcium Oxalate Crystal Induced Reactive Oxygen Species in Kidneys. J. Urol. 2015, 193, 1684-1691.

[30] Ligon, C. B.; Hummers, L. K.; McMahan, Z. H. Oxalate nephropathy in systemic sclerosis: Case series and review of the literature. Semin. Arthritis Rheum. 2015, 45, 315-320.

[31] Veena, C. K.; Josephine, A.; Preetha, S. P.; Rajesh, N. G.; Varalakshmi, P. Mitochondrial ysfunction in an animal model of hyperoxaluria: A prophylactic approach with fucoidan. Eur. J. Pharmacol. 2008, 579, 330-336.
[32] Bayir, Y.; Halici, Z.; Keles, M.S.; Colak, S.; Cakir, A.; Kaya, Y.; Akçay, F. Helichrysum plicatum DC. subsp. plicatum extract as a preventive agent in experimentally induced urolithiasis model. Ethnopharmacology 2011, 138, 408-414.

[33] Bhadja, P.; Lunagariya, J.; Ouyang, J. M. Seaweed sulphated polysaccharide as an inhibitor of calcium oxalate renal stone formation. J. Funct. Foods 2016, 27, 685-694

[34] Ratkalkar, V. N.; Kleinman, J. G. Mechanisms of Stone Formation. Clin. Rev. Bone Miner. 2011, 9, 187-197.

Received November 27, 2019

Accepted April 14, 2020 